\title{
ISAR-REACT 5 trial: a new guidance for the treatment of acute coronary syndromes
}

\author{
ISAR-REACT 5 trial: una nueva guía para el tratamiento \\ de los síndromes coronarios agudos
}

Revista Argentina de Cardioangiología Intervencionista 2019;10(3):108-110. https://doi.org/10.30567/RACI/201903/0108-0110

In the last 10 years clinicians have been faced with the dilemma which platelet P2Y12 receptor antagonist to choose for the treatment of patients with acute coronary syndrome (ACS). This is because two P2Y12 receptor antagonists, prasugrel $^{1}$ and ticagrelor ${ }^{2}$, had shown superior efficacy over their predecessor, clopidogrel, in large scale randomized clinical trials and received a class I recommendation in the guidelines. ${ }^{3}$ The decision making process was further complicated by the fact that, according to guidelines, the 2 new drugs were to be administered in 2 different ways in patients with non-ST-segment elevation ACS (NSTE-ACS). Available evidence supports routine initiation of ticagrelor as soon as the diagnosis of NSTE-ACS was established without waiting for the results of diagnostic angiography. ${ }^{2,3}$ The recommendation is different for prasugrel. In patients with NSTE-ACS, initiation of prasugrel was dependent on the findings of diagnostic angiography. ${ }^{1,3}$ The reason behind are the results of a specifically designed trial that showed that pretreatment with prasugrel was not only not beneficial but also harmful in patients with NSTE-ACS. ${ }^{4}$ The lack of head to head comparison trials of ticagrelor vs. prasugrel given over one year for the whole spectrum of ACS made difficult the decision of whether to embrace a ticagrelor-based strategy with routine pretreatment or a prasugrel-based strategy that requires waiting for the results of diagnostic angiography before starting prasugrel treatment in patients with NSTE-ACS.

The Intracoronary Stenting and Antithrombotic Regimen: Rapid Early Action for Coronary Treatment (ISAR-REACT) 5 trial was an investigator-initiated, randomized, multicenter, open label trial that compared a ticagrelor- with a prasugrel-based strategy in 4,018 patients. ${ }^{5,6}$ The study hypothesis was that ticagrelor is superior to prasugrel. However, contrary to our expectation, the primary endpoint of death, myocardial infarction, or stroke at one year was observed significantly more frequently with the ticagrelor-based strategy $(9.3 \%$ in the ticagrelor group $6.9 \%$ in the prasugrel group, hazard ratio, 1.36; $95 \%$ confidence interval [CI], 1.09 to $1.70 ; \mathrm{P}=0.006$, Figure 1 ). The superiority of prasugrel was consistent across the ACS subsets irrespective of whether the patients presented with ST-segment elevation myocardial infarction (STEMI) or NSTE-ACS. Importantly, the increased efficacy of prasugrel (26\% reduction in the risk of the primary endpoint) did not occur at the expense of an increased bleeding risk. Major bleeding (defined as Bleeding Academic Research Consortium (BARC) type 3-5) was observed in 5.4\% of patients in the ticagrelor group and in $4.8 \%$ of patients in the prasugrel group (hazard ratio, $1.12 ; 95 \% \mathrm{CI}, 0.83$ to $1.51 ; \mathrm{P}=0.46$; Figure 1 ). ${ }^{6}$ The ISAR-REACT 5 trial provides two main lessons: Firstly, prasugrel is a more effective antiplatelet drug than ticagrelor in ACS patients. The best clue to this is provided by the subset of STEMI patients in whom pretreatment was the starting strategy for both study drugs. Secondly, pretreatment with ticagrelor does not offer any advantage in patients with NSTE-ACS. Although there was no study arm without ticagrelor pretreatment in the ISAR-REACT 5 trial, taken together, our trial and the A Comparison of prasugrel at the time of percutaneous Coronary intervention Or as pre-treatment At the time of diagnosis in patients with non-ST-segment elevation myocardial infarction (ACCOAST) trial offer evidence that pretreatment is not needed in this subset of ACS patients.

In consideration of the feedback received after the publication of the primary results ${ }^{6}$, several aspects of the ISAR-REACT 5 trial need to be discussed in more detail.

First, a double-blind, placebo-controlled design is an undisputed strength of a randomized trial. The ISAR-REACT 5 trial started to recruit patients in a time in which both study drugs, ticagrelor and prasugrel, were patented and no generic drugs were available (a generic ticagrelor does still not exist). Provision of placebo tablets is not only a cost-increasing factor but also usually impossible without the involvement of the study drug manufacturing companies. The investigators of the ISAR-REACT 5 trial failed in their efforts to obtain the support of these companies and there was no alternative left to the open-label design. However, the excellent comparability of baseline characteristics, the blinded adjudication of all events that composed the primary and secondary endpoints and a final result that did not confirm the study hypothesis argue against a relevant bias related to the open label design in the ISAR-REACT 5 trial. Second, the design of the ISAR-REACT 5 trial was guided by both, current guidelines and practice. While in STEMI patients treatment strategies were the same (knowledge of coronary anatomy was not required for initiation of therapy in either study arm), patients with NSTE-ACS were routinely pretreated only if they were assigned to the ticagrelor arm. In patients with NSTE-ACS belonging to the prasugrel arm, administration of the loading dose was dependent on the findings of diagnostic angiography. This explains the lower number of patients in the prasugrel group that received the first loading dose as compared with those in the ticagrelor group. In line with current practice, pa- 


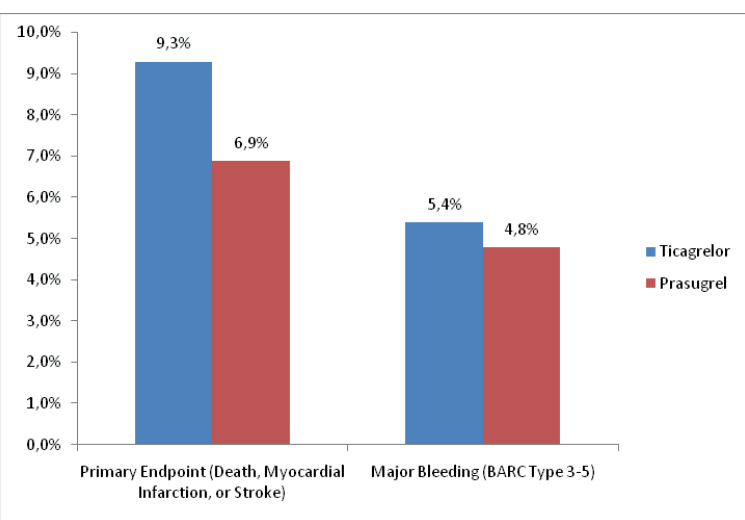

Figure 1. Cumulative incidence of the primary endpoint, the composite of death, myocardial infarction, or stroke and the safety endpoint of bleeding BARC type 3-5 at 12 months after randomization in patients assigned to ticagrelor and prasugrel. tients who did not show obstructive coronary artery disease in coronary angiography and those who resulted to have an indication of oral anticoagulation after randomization were not discharged on the study drug. In addition, $15.2 \%$ of the patients assigned to ticagrelor and $12.5 \%$ of the patients assigned to prasugrel discontinued study therapy at some time after discharge up to one-year follow-up. ${ }^{6}$ Higher discontinuation rates for ticagrelor as compared to the control drug have been a consistent finding of many randomized clinical trials on ticagrelor. ${ }^{2,78}$ An analysis based on the intention-to-treat principle, as it was done for the primary endpoint in the ISAR-REACT 5 trial, is considered the gold standard for the assessment of efficacy, is also recommended by regulatory agencies and has virtually been used in all pivotal randomized clinical trials on antithrombotic therapies. For example, an intention to treat analysis was also used in the Prevention of Cardiovascular Events in Patients with Prior Heart Attack Using Ticagrelor Compared to Placebo on a Background of Aspirin-Thrombolysis in Myocardial Infarction 54 (PEGASUS-TIMI 54) trial, in which 32\% of patients assigned to $90 \mathrm{mg}$ ticagrelor discontinued the drug prematurely.? Third, a positive finding of the ISAR-REACT 5 trial was that the enhanced efficacy with prasugrel was not achieved at the expense of an increased risk of bleeding. This finding came at a surprise to some colleagues who always expect an inverse correlation in the response of ischemic and bleeding complications to antithrombotic drugs. However, there are many examples that show that this is not the the rule. Indeed, the first ISAR trial showed that an increase in efficacy can be achieved by replacing anticoagulation with antiplatelet drugs even with a reduction in bleeding. ${ }^{9}$ Other examples challenging this paradigm include the pivotal trials comparing NOACs with vitamin $\mathrm{K}$ antagonists. ${ }^{10}$ More recently, several trials on de-escalation treatment strategies have successfully shown reduction in bleeding while efficacy was maintained. ${ }^{11}$ In this regard, the reduction of the prasugrel maintenance dose from 10 to $5 \mathrm{mg}$ daily in high risk bleeding patients (age $\geq 75$ years or bodyweight below $60 \mathrm{~kg}$ ) may have contributed to the improvement of safety with prasugrel in the ISAR-REACT 5 trial while all patients in the ticagrelor group received $90 \mathrm{mg}$ bid without dose individualization.

Fourth, the $26 \%$ reduction in the risk of ischemic complication with prasugrel as compared to ticagrelor in the ISAR-REACT 5 trial has also been put in the context of the findings of previous pivotal placebo-controlled trials with antiplatelet drugs in ACS patients. In the Clopidogrel in Unstable angina to prevent Recurrent Events (CURE) trial $^{12}$, clopidogrel was associated with a $20 \%$ reduction in the composite of cardiovascular death, myocardial infarction, or stroke compared to placebo. However, there are major differences between the CURE and ISAR-REACT 5 trials, with a time interval of more than 15 years between them. Only $21 \%$ of the patients enrolled in the CURE trial underwent a PCI procedure as compared with $84 \%$ of the patients in the ISAR-REACT 5 trial. In fact, the treatment effect of clopidogrel was $30 \%$ when only the PCI patients were evaluated in the CURE trial. ${ }^{13}$ Moreover, all PCI patients in the CURE trial received clopidogrel for 4 weeks after PCI (the period with the highest event rate), irrespective of the arm to which they were assigned to at enrolment. In the clopidogrel arm of the CURE trial, clopidogrel was given for 3 to 12 months (mean of only 8 months). Thus, the design of the CURE trial does not allow to assess the maximal treatment effect of clopidogrel in these patients.

We do not know the exact mechanisms of the advantage of prasugrel over ticagrelor in ACS patients as shown in the ISAR-REACT 5 trial. Although both of them are P2Y12 receptor antagonists, they differ from each other regarding their chemical structure, reversibility of antagonism, dosage frequency, pleiotropic effects, side effect profile, drugdrug interactions and drug-compliance. One or a combination of these factors may have driven the trial results in favor of prasugrel. Although elucidation of the underlying mechanism(s) is an important goal to pursue, the limited knowledge in this regard does not reduce the paradigm changing value of the ISAR-REACT 5 trial.

A few major characteristics of the ISAR-REACT 5 trial should be taken into consideration before extrapolating its results to the everyday practice. Notably, the study population in this trial is characterized by a very high proportion of patients treated with PCI and a very low proportion of patients treated with CABG or conservatively. Having said that, the results of the ISAR-REACT 5 trial are going to simplify the antiplatelet treatment algorithm of patients with ACS, making of prasugrel - in an individualized dose regimen - the mainstay of this treatment in all cases without specific contraindications to its use. While the diagnosis of STEMI is relatively straightforward and allows for starting prasugrel at the time of admission, in all patients with NSTE-ACS, initiation of prasugrel is dependent on the findings of diagnostic angiography.

Stefanie Schüpke, Adnan Kastrati

ISAResearch Center, Deutsches Herzzentrum München, Technische Universität München and DZHK (German Center for Cardiovascular Research), Partner Site Munich Heart Alliance 


\section{REFERENCES}

1. Wiviott SD, Braunwald E, McCabe CH, et al. Prasugrel versus clopidogrel in patients with acute coronary syndromes. NEngl J Med 2007;357:2001-15.

2. Wallentin L, Becker RC, Budaj A, et al. Ticagrelor versus clopidogrel in patients with acute coronary syndromes. N Engl J Med 2009;361:1045-57.

3. Neumann FJ, Sousa-Uva M, Ahlsson A, et al. 2018 ESC/EACTS Guidelines on myocardial revascularization. Eur Heart J 2019:40:87-165.

4. Montalescot G, Bolognese L, Dudek D, et al. Pretreatment with prasugrel in non-ST-segment elevation acute coronary syndromes. N Engl J Med 2013;369:999-1010.

5. Schulz S, Angiolillo DJ, Antoniucci D, et al. Randomized comparison of ticagrelor versus prasugrel in patients with acute coronary syndrome and planned invasive strategy--design and rationale of the iNtracoronary Stenting and Antithrombotic Regimen: Rapid Early Action for Coronary Treatment (ISAR-REACT) 5 trial. J Cardiovasc TransI Res 2014;7:91-100.

6. SchupkeS, Neumann FJ, Menichelli M, et al. Ticagrelor or Prasugrel in Patients with Acute Coronary Syndromes. N Engl J Med 2019.

7. Bonaca MP, Bhatt DL, Cohen M, et al. Long-term use of ticagrelor in patients with prior myocardial infarction. N Engl J Med 2015;372:1791-800.

8. Hiatt WR, Fowkes FG, Heizer G, et al. Ticagrelorversus Clopidogrel in Symptomatic Peripheral Artery Disease. N Engl J Med 2017;376:32-40.

9. Schomig A, Neumann FJ, Kastrati A, et al. A randomized comparison of antiplatelet and anticoagulant therapy after the placement of coronary-artery stents. N Engl J Med 1996;334:1084-9.

10. Ruff CT, Giugliano RP, Braunwald E, et al. Comparison of the efficacy and safety of new oral anticoagulants with warfarin in patients with atrial fibrillation: a meta-analysis of randomised trials. Lancet 2014;383:955-62.

11. Mehran R, Baber U, Sharma SK, et al. Ticagrelor with or without Aspirin in High-Risk Patients after PCI. N Engl J Med 2019

12. YusufS, Zhao F, Mehta SR, Chrolavicius S, Tognoni G, Fox KK. Effects of clopidogrel in addition to aspirin in patients with acute coronary syndromes without ST-segment elevation. N Engl J Med 2001;345:494-502.

13. Mehta SR, Yusuf S, Peters RJ, et al. Effects of pretreatment with clopidogrel and aspirin followed by long-term therapy in patients undergoing percutaneous coronary intervention: the PCI-CURE study. Lancet 2001;358:527-33. 